Review Article

\title{
Fragile X syndrome, the search for a targeted treatment
}

\author{
S. Zeidler*, R. Willemsen \\ Department of Clinical Genetics, Erasmus MC, Rotterdam, The Netherlands
}

Article Info Abstract

History

Received : 17 Dec 2018

Accepted : 23 March 2019

Available : 27 July 2019

\begin{abstract}
Background : Fragile X syndrome (FXS), the most common monogenetic cause of intellectual disability and autism spectrum disorders, is characterized by behavioral and physical problems. There is currently no adequate treatment available. While animal model studies seemed extremely promising, no success has been achieved in the larger clinical trials with human FXS patients. This short review describes the steps that have been taken in the development of a targeted treatment for FXS. Possible reasons for the lack of translation between animal models and human FXS patients are being explored and solutions are being proposed. The FXS story illustrates pitfalls and possibilities in translational research, that might especially be applicable for other neurodevelopmental disorders as well
\end{abstract}

Keywords FMR1, fragile X syndrome, Fmr1 KO mouse, mGluR5, GABA, clinical trial, outcome measure

Permalink/ DOI: https://doi.org/10.14710/jbtr.v5i1.3925

\section{INTRODUCTION}

Fragile X syndrome (FXS) is the most common monogenetic cause of intellectual disability and autism spectrum disorders, affecting about 1:7000 males ${ }^{1,2}$. The disorder is caused by a CGG repeat expansion in the 5, UTR of the FMRl gene. This repeat expansion leads to silencing of the FMRI gene and lack of its protein product, FMRP. Since FMRP plays an important role in regulation of synaptic plasticity in the brain, its lack leads to several neurocognitive and behavioral problems. Hence, FXS is accompanied by intellectual disability, autism spectrum disorders, executive function deficits, attention and hyperactivity disorder, aggression and anxiety, amongst others. Also medical problems are frequent, including epilepsy and frequent otitis media in children ${ }^{3,4}$. Patients are usually attending special education or end up in institutions. Emotional and behavioral problems are most disabling and are often treated with non-specific symptomatic pharmacological and supportive treatments. However, these interventions are mostly insufficient and there is no disease-modifying effective therapy, targeting the cognitive, behavioral, emotional or medical problems.

\footnotetext{
* Corresponding author:

E-mail: s.zeidler@erasmusmc.nl (S. Zeidler)
}

This lack of effective therapy leaves parents, care-givers and medical professionals with little options to alleviate the burden of taking care of a patient with FXS. Moreover, its relatively high frequency together with the life-long intellectual, and sometimes extreme behavioral and physical disabilities and the hereditary character of the disease, make FXS very costly for society. Hence, an effective targeted disease modifying therapy, especially affecting the behavioral, emotional and intellectual problems, is important for patients, caregivers, physicians and society.

\section{THE SEARCH FOR A TARGETED TREATMENT}

Identification of the FMRl gene as the causative gene ${ }^{5}$, opened possibilities to study the disease in animal models. In the past decades, much research has been performed on FXS animal models, for example the Fmrl knock-out (KO) mouse, the fruit fly and the zebrafish. This research identified the function of the FMR 1 protein product, FMRP, as a key regulator of the neuronal synaptic plasticity, by binding and regulating the local translation of target mRNAs. Many synaptic pathways have been shown to be disturbed in $\mathrm{FXS}^{6}$. A few of these include the metabotropic glutamate type 5 receptor (mGluR5) pathway, the gamma-Aminobutyric acid (GABA)ergic pathway, the endocannabinoid pathway, the matrix metalloproteinase 9 (MMP9) pathway and intracellular signaling pathways (e.g. extracellular signal related kinase (ERK), mammalian target of rapamycin 
(mTOR) and glycogen synthase kinase-3 (GSK3)). Also intracellular downstream mRNA and protein targets of FMRP have been shown to be involved ${ }^{6,7}$. All these pathways are part of the immensely complex network, balancing the neurons' excitatory and inhibitory function and thus regulating synaptic plasticity.

Since the identification of the pathways involved in FXS, many studies have focused on preclinical research targeting the aberrant pathways in FXS animal models, leading to promising therapeutic results ${ }^{6,8}$. While FXS initially seemed to be a complex and untreatable disease, it unexpectedly held promise of a therapy. Enthusiastically, clinical studies with FXS patients followed, targeting several pathways, including the mGluR5 pathway, GABAergic pathway, MMP9 and GSK3 ${ }^{6,7}$. Although some of the smaller, controlled and non-controlled trials seemed to yield some improvement in behavior, none of the larger, placebo-controlled trials have met their endpoints and proved efficacious in FXS patients ${ }^{9}$. What is the reason that the promising animal studies could not be translated into a functional therapy for humans? What went wrong?

\section{POSSIBLE REASONS AND SOLUTIONS FOR THE LACK OF TRANSLATION}

Clearly some issues need to be addressed, before the FXS research field can continue its search for a therapy. One of the major question is: are animal models suitable in modeling the human disease in the search of a therapy? Although animal models are indispensable in studying human diseases and in preclinical assessment of treatment, there are of course important differences between animals and humans. The Fmrl KO mouse shows many similarities to the human disease, including macro-orchidism, behavioral and cognitive features ${ }^{10}$. However, many of the phenotypes are subtle in the mouse model. Moreover, publications have shown great differences in their results, which is possibly accountable for by different genetic backgrounds and the setup of extreme controlled laboratory experimental protocols 10,11. Results from studies in only one animal model cannot be generalized to humans ${ }^{12}$. Possibly, reproducing successful results in several genetic backgrounds of several different animal species, could more precisely predict a chance for successful translation.

Another important limitation of the FXS studies are the used outcome measures, in mice as well as in humans 6,13 . To determine an effect of treatment, changes of relevant and important phenotypes should be used as outcome measures. However, many of the outcome measures used in animal models are probably not, or poorly, translatable to human functioning. In addition, biochemical measures, electrophysiological and microscopic morphological measures are often difficult to translate into relevant clinically functional improvement. In the clinical trial setting, most used outcome measures were care-giver rated questionnaires. Those are very prone to large placebo effects and are not objective. Currently, efforts are ongoing in the development of relevant and more objective outcome measures, that preferably should be usable in humans as well as in animal models. When using questionnaires, those should be validated for disease modifying therapy and more extensive training of the caregiver is needed to secure reliable results. Also, more objective, relevant and accessible outcome measures should be explored in their suitability for clinical trials, including endophenotypes like the eye-blink test, biomarkers, functional MRI (fMRI), functional near-infrared spectroscopy (fNIRS), Transcranial Magnetic Stimulation (TMS) and electroencephalogram (EEG).

The trial design could pose another important limitation in FXS studies. In fact, current trial design is probably not suitable to investigate disease modifying treatments for rare diseases, especially in the case of neurodevelopmental disorders. Typically, for safety and ethical reasons studies are not performed in very young children and are often carried out for a short period of time. However, in neurodevelopmental disorders treatment from a very young age should probably be aspired, as well as a longer duration of treatment. Recently, a trial has been initiated treating young children with the mGluR5-antagonist mavoglurant (NCT02920892). Additionally, drug treatment should be combined with environmental enrichment, to secure controlled stimulation of synaptic plasticity ${ }^{14}$.

Finally, the least addressed but probably most important issue, concerns the need to target more than one pathway simultaneously. Considering the large number of pathways and downstream mRNA's and proteins involved, it is highly unlikely that targeting only one of these would be enough to ameliorate the disorder 11. Only a few studies have focused on combination therapy, targeting more than one pathway, including studies targeting the mGluR5 and GABAergic pathway simultaneously ${ }^{15,16}$ and the serotonin and dopamine pathways simultaneously ${ }^{17}$. These studies do support the theory that those different pathways are interconnected in a delicate balance. However, a tremendous fine-tuning will presumably be needed to restore the synaptic plasticity at the individual level. Theoretically, the most effective treatment would be to reactivate endogenous transcription of the FMRI gene, preferably at a young age. Recently, efforts to reactivate transcription have been made successfully in cell models, using CRISPR/Cas9 and small molecules ${ }^{18,19}$. However, these interventions are currently far from applicable in the clinical setting.

\section{CONCLUSION}

While the quest for a targeted treatment for FXS is still ongoing, lessons that have been learned so far are being addressed in new approaches. These lessons are not only relevant for FXS, but could be generalized for all neurodevelopmental disorders for which a disease modifying therapy is being developed, or even translational medicine in general. Studies are performed exploring possibilities in the use of other disease models (e.g. other animal models, organoids and organ on a chip), outcome measures and alternative trial design. Tackling the complex issue of combination therapy is probably even more challenging, and should be considered in future studies. Perhaps, in the near future, successful and safe reactivation of transcription of the FMRI gene will be feasible, opening new possibilities. 


\section{REFERENCES}

1. Hunter J, Rivero-Arias O, Angelov A, Kim E, Fotheringham I, Leal J: Epidemiology of fragile $X$ syndrome: a systematic review and meta-analysis. Am J Med Genet A 2014; 164A: 1648-1658.

2. Rauch A, Hoyer J, Guth $S$ et al: Diagnostic yield of various genetic approaches in patients with unexplained developmental delay or mental retardation. Am J Med Genet A 2006; 140: 2063 2074.

3. Hersh JH, Saul RA, Committee on G: Health supervision for children with fragile $X$ syndrome. Pediatrics 2011; 127: 994-1006.

4. Kidd SA, Lachiewicz A, Barbouth $D$ et al: Fragile $X$ syndrome: a review of associated medical problems. Pediatrics 2014; 134: 995-1005.

5. Verkerk AJ, Pieretti $M$, Sutcliffe JS et al: Identification of a gene (FMR-1) containing a CGG repeat coincident with a breakpoint cluster region exhibiting length variation in fragile $X$ syndrome. Cell 1991; 65: 905-914.

6. de Esch CE, Zeidler S, Willemsen R: Translational endpoints in fragile $X$ syndrome. Neurosci Biobehav Rev 2014; 46 Pt 2: 256-269.

7. Gross C, Hoffmann A, Bassell GJ, Berry-Kravis EM: Therapeutic Strategies in Fragile $X$ Syndrome: From Bench to Bedside and Back. Neurotherapeutics 2015; 12: 584-608.

8. Ligsay A, Hagerman RJ: Review of targeted treatments in fragile $X$ syndrome. Intractable Rare Dis Res 2016; 5: 158-167.

9. Berry-Kravis EM, Lindemann L, Jonch AE et al: Drug development for neurodevelopmental disorders: lessons learned from fragile $\mathrm{X}$ syndrome. Nat Rev Drug Discov 2018; 17: 280-299.

10. Kazdoba TM, Leach PT, Silverman JL, Crawley JN: Modeling fragile $X$ syndrome in the Fmr1 knockout mouse. Intractable Rare Dis Res 2014; 3: 118-133.
11. Zeidler S, Hukema RK, Willemsen R: The quest for targeted therapy in fragile $X$ syndrome. Expert Opin Ther Targets 2015; 19: 1277-1281.

12. van der Worp HB, Howells DW, Sena ES et al: Can animal models of disease reliably inform human studies? PLoS Med 2010; 7: e1000245.

13. Budimirovic DB, Berry-Kravis E, Erickson CA et al: Updated report on tools to measure outcomes of clinical trials in fragile $\mathrm{X}$ syndrome. J Neurodev Disord 2017; 9: 14.

14. Castren E, Elgersma Y, Maffei L, Hagerman R: Treatment of neurodevelopmental disorders in adulthood. J Neurosci 2012; 32: 14074-14079.

15. Zeidler S, de Boer $H$, Hukema RK, Willemsen R: Combination Therapy in Fragile $X$ Syndrome; Possibilities and Pitfalls Illustrated by Targeting the mGluR5 and GABA Pathway Simultaneously. Front Mol Neurosci 2017; 10: 368.

16. Pacey LK, Tharmalingam S, Hampson DR: Subchronic administration and combination metabotropic glutamate and $G A B A B$ receptor drug therapy in fragile X syndrome. J Pharmacol Exp Ther 2011; 338: 897-905.

17. Lim CS, Hoang ET, Viar KE, Stornetta RL, Scott MM, Zhu JJ: Pharmacological rescue of Ras signaling, GluA1-dependent synaptic plasticity, and learning deficits in a fragile X model. Genes Dev 2014; 28: 273-289.

18. Kraan CM, Godler DE, Amor DJ: Epigenetics of fragile $\mathrm{X}$ syndrome and fragile X-related disorders. Dev Med Child Neurol 2019; 61: 121-127.

19. Yrigollen CM, Davidson BL: CRISPR to the Rescue: Advances in Gene Editing for the FMR1 Gene. Brain Sci 2019; 9. 